\title{
Uplink Cell-Free Massive MIMO-NOMA Systems Based on Group-Level Successive Interference Cancellation
}

\section{Tao Cui ( $D$ 826865293@qq.com )}

Nanjing University of Posts and Telecommunications https://orcid.org/0000-0002-3695-2439

Chengcheng Liu

Nanjing University of Posts and Telecommunications

Peng Du

Nanjing University of Posts and Telecommunications

Rongfang Song

Nanjing University of Posts and Telecommunications

\section{Research Article}

Keywords: Cell-free massive MIMO, on-orthogonal multiple access, group-level successive interference cancellation, power control

Posted Date: January 18th, 2022

DOI: https://doi.org/10.21203/rs.3.rs-1257395/v1

License: (c) (i) This work is licensed under a Creative Commons Attribution 4.0 International License. Read Full License 


\section{Uplink Cell-Free Massive MIMO-NOMA Systems Based on Group-Level Successive Interference Cancellation}

Tao Cui ${ }^{1}$, Chengcheng Liu ${ }^{1}$, Peng Du ${ }^{1,2}$ and Rongfang Song ${ }^{1 *}$

\author{
${ }^{*}$ Correspondence: \\ songrf@njupt.edu.cn \\ ${ }^{1}$ Key Lab of Broadband Wireless \\ Communication and Sensor \\ Network Technology, Nanjing \\ University of Posts and \\ Telecommunications, Nanjing, \\ China \\ Full list of author information is \\ available at the end of the article
}

\begin{abstract}
Non-orthogonal multiple access (NOMA) and multiple-input multiple-output (MIMO) are viewed as promising techniques to meet the demands for high spectrum efficiency and massive connectivity in future wireless communication. In this paper, a novel framework for realizing transmission in cell-free massive MIMO-NOMA system with deep integration between MIMO and NOMA is proposed. A new method is developed to divide users into different groups according to their equivalent path loss, then "group-level successive interference cancellation" (GSIC) is used to cancel inter-group interference when demodulating users. Based on the new framework, a rigorous closed-form expression of achievable sum rate in uplink cell-free massive MIMO-NOMA system is derived. Besides, a parallel iterative method is used to obtain the best power control scheme. It is shown by simulations that the proposed scheme can effectively reduce total power consumption and outperforms the orthogonal multiple access (OMA) transmission and the traditional SIC-NOMA schemes based on user clustering.
\end{abstract}

Keywords: Cell-free massive MIMO; on-orthogonal multiple access; group-level successive interference cancellation; power control

\section{Introduction}

With the ever-increasing demands for massive connectivity and high spectrum efficiency for the upcoming beyond fifth generation $(\mathrm{B} 5 \mathrm{G})$ and the sixth generation (6G) networks, non-orthogonal multiple access (NOMA) and massive multiple-input multiple-output (MIMO) are viewed as key techniques [1]. NOMA mainly includes two categories: power domain NOMA and code domain NOMA. This paper focuses on the former. In the power domain NOMA, it transmits multiple user messages on the same resource blocks. At the receiver, the demodulation order of multiple users is determined by channel state information (CSI) and successive interference cancellation (SIC) technique is used to remove inter-user interference [2], hence the system capacity can be significantly increased. However, in the system of combining NOMA with MIMO (MIMO-NOMA), user channels are in the form of matrices, which fails to determine the demodulation order for users during SIC based on CSI. Thus, the research for massive MIMO-NOMA becomes more challenging.

\subsection{Motivations and Contributions}

In the existing studies for MIMO-NOMA, there are mainly two categories: userclustered method and user-specific beamforming method. In [3], the signal alignment 
method was used to optimize the MIMO-NOMA system. First, users with large path loss differences were randomly divided into a cluster, then users in the same cluster were aligned to a direction through precoding. The inter-cluster interference was suppressed by base station equalizer, while SIC was used to remove intra-cluster interference. However, user-clustered method based on signal alignment has the situation that the angle between the equivalent channels after signal alignment may be very small, for which some useful signals will be lost when the base station equalizer is used to suppress inter-cluster interference, causing the problem of high power consumption. User-specific beamforming method was used in [4]. Differing from user-clustered method, each user in this method had a different beamforming vector so that the optimization degree of freedom was obviously greater than that of the user-clustered method. However, this method failed to reflect the advantages of space division multiplex access (SDMA) in MIMO-NOMA, which means SIC must be used among all users occupying the same resource blocks. Once a user has error decoding, serious error propagation will occur, which greatly influences the performance of the system. In view of the limitations of the above mentioned works, in [5], a precoding scheme based on the novel grouping was proposed in uplink MIMO-NOMA systems, which made attempt to group-level optimization. The group-level successive interference cancellation (GSIC) was proposed in reflecting intelligent surface (RIS) aided uplink NOMA system for the first time in [6]. Users were divided into different groups according to CSI and the users in the same group were considered as a whole. The GSIC was used to remove inter-group interference by sequentially demodulating group users, while orthogonal multiple access (OMA)/SDMA was used among intra-group users.

This paper proposes to apply GSIC to the uplink cell-free massive MIMO-NOMA systems by dividing users into different groups based on their differences in equivalent path loss. Through the transmission of NOMA among different groups and SDMA within each groups, the transmission mechanism of deep integration between MIMO and NOMA is realized. Unlike traditional cellular networks, cell-free massive MIMO cancels the concept of cells, which enables its ability to avoid the inter-cell interference [7]. A large number of distributed access points (APs) jointly provide uniform service to all users using the same resource blocks [8]. Besides, a central processing unit (CPU) is designed to connect all APs through backhaul network. Then, simple signal processing is performed in each AP and CPU conducts complex processing. Cell-free massive MIMO-NOMA system was studied in [9, 10, 11]. In [9], the authors took the first attempt to combine NOMA with cell-free massive MIMO and derived the achievable sum rate with imperfect SIC. In [10], a low complexity sub-optimal user-clustered method based on Jaccord coefficients was proposed to improve the sum rates of cell-free massive MIMO-NOMA system. A spectral efficiency maximization scheme with a low complexity algorithm for cell-free massive MIMO-NOMA system was studied in [11]. The above works were based on user-clustered method and the users in the same cluster followed the principle of NOMA, while the users among different clusters followed the principle of SDMA. Just like in single cell MIMO-NOMA system, above clustering based scheme has the disadvantage of low power efficiency. The combination of GSIC and cell-free massive MIMO-NOMA is expected to improve the power efficiency significantly. 
Motivated by this, this paper focuses on the uplink performance of cell-free massive MIMO-NOMA based on GSIC. Our contributions are listed as follows:

(1) A novel framework of group-level optimization based on GSIC is proposed for the uplink cell-free massive MIMO-NOMA system, in which users are divided into different groups based on their equivalent path loss for the first time. In the new framework, the inter-group multiple access follows the principle of NOMA and GSIC is invoked to reduce the inter-group interference, while the intra-group multiple access follows the principle of SDMA. Besides, an achievable sum rate is derived under the consideration of error propagation and intra-group interference.

(2) A power minimization problem is formulated on the premise of ensuring per user's quality of service (QoS) and transmit power limit. Using the relationship between the equalizers and the transmit power coefficients, the original joint optimization problem is transformed to power optimization problem. Besides, the optimal power control scheme is obtained through a parallel iterative method. It is shown by simulations that compared with OMA and SIC-NOMA based on user clustering, the proposed scheme can effectively reduce the total power consumption. It is worth mentioning that the effect is significant when the number of users is large.

\subsection{Notations}

Throughout this paper, lower-case boldface letter denotes vector and matrix is represented by upper-case boldface letter. $\mathbf{z}^{H}, \mathbf{z}^{*}$, and $[\mathbf{z}]_{k}$ denote the conjugatetranspose, conjugate, and the $k$ th element of a vector $\mathbf{z}$, respectively. $|\cdot|$ indicates absolute operator. Finally, $\mathbf{n} \sim \mathcal{C} \mathcal{N}(0,1)$ means a complex Gaussian random vector, whose mean is zero and variance is 1 .

\section{Methods}

\subsection{System Model}

In this paper, the uplink transmission of cell-free massive MIMO-NOMA system is considered in which $K$ users and $L$ APs equipped with single-antenna are randomly and uniformly distributed in a square area. All APs are connected to a CPU through backhaul network. The users are divided into $M$ groups based on their equivalent path loss to all APs and the number of users in a group is $U_{k}$. For convenience, the $k$-th user in the $m$-th group is defined as $\mathrm{UE}_{\mathrm{m}, \mathrm{k}}$. Moreover, the channel gain between $\mathrm{UE}_{\mathrm{m}, \mathrm{k}}$ and all APs can be modeled as

$$
\mathbf{h}_{m, k}=\boldsymbol{\beta}_{m, k}^{1 / 2} \mathbf{g}_{m, k}
$$

with

$$
\boldsymbol{\beta}_{m, k}=\operatorname{diag}\left\{\beta_{1, m, k}, \cdots, \beta_{l, m, k}, \cdots, \beta_{L, m, k}\right\},
$$

where $\boldsymbol{\beta}_{m, k}$ is a diagonal matrix and $\beta_{l, m, k}$ denotes the large-scale channel gain which changes very slowly. Besides, each element in $\mathbf{g}_{m, k}$ follows a complex Gaussian distributed with zero mean and unit variance independently. 


\subsubsection{New method of user grouping}

The so-called GSIC refers to that the users are divided into different groups following some principle and the users in a group is considered as a whole, which can also be regared as a virtual user. After sorting the groups (virtual users), the virtual users can be sequentially demodulated and the inter-group interference can be removed.

To divide the users into multiple groups, this paper puts forward a new method of user grouping based on per user's equivalent path loss. The users in different groups follow the principle of NOMA, using GSIC to remove the inter-group interference, while the users in the same group follow the principle of SDMA. Therefore, two factors taken into account when user grouping are as follows:

(i) The number of users in each group: Since the users in the same group follow the principle of SDMA, the number of users in each group should be less than the number of APs, i.e., $\mathrm{U}_{\mathrm{k}}<L$, which ensures the users in each group can be demodulated in parallel.

(ii) The equivalent pass loss of per user: In a single-cell massive MIMO-NOMA system, users can be divided into different groups according to their path loss to the base station. Motivated by this, we propose a new method of user grouping in cell-free massive MIMO-NOMA system. Since users are served by all APs, user grouping can be implemented in the light of per user's equivalent path loss to all APs. That's to say, users with the same equivalent path loss are divided into a group. The equivalent path loss of $\mathrm{UE}_{\mathrm{m}, \mathrm{k}}$ to all APs is defined as follows:

$$
\lambda_{m, k}=\frac{1}{\sum_{l=1}^{L} \beta_{l, m, k}}(m=1,2, \cdots, M, k=1,2, \cdots, K),
$$

where $\beta_{l, m, k}$ captures the large-scale channel gain of $\mathrm{UE}_{\mathrm{m}, \mathrm{k}}$. The equivalent path loss of users in different groups should meet the the following equation:

$$
\lambda_{m, k}<\lambda_{m^{\prime}, k^{\prime}}\left(m<m^{\prime}\right)
$$

We present an example of equivalent path loss contours for five APs in Fig.1, in which users with same equivalent pass loss are in the same contour.

\section{DGX}

Figure 1 llustration of equivalent path loss contour for a cell-free system with five APs.

\subsubsection{Uplink Data Transmission}

In the uplink transmission, user messages are transmitted to all APs simultaneously. The received signal at the $l$-th AP can be expressed as:

$$
\mathbf{f}=\sum_{m=1}^{M} \sum_{k=1}^{\mathrm{U}_{k}} \mathbf{h}_{m, k} w_{m, k} x_{m, k}+\mathbf{v}
$$

where $\mathbf{h}_{m, k}$ denotes channel gain vector between $\mathrm{UE}_{\mathrm{m}, \mathrm{k}}$ and $l$-th $\mathrm{AP}, w_{m, k}$ and $x_{m, k}$ represent the transmit power coefficient and transmit signal of $\mathrm{UE}_{\mathrm{m}, \mathrm{k}}$, respectively. 
Besides, $\mathbf{v}$ is the noise vector with each element following $\mathcal{C N}\left(0, \sigma_{m, k}^{2}\right)$. In order to facilitate the derivation of the uplink achievable sum rate formula, we rewrite the received signal at the $l$-th $\mathrm{AP}$ in the form of a matrix:

$$
\mathbf{f}=\sum_{m=1}^{M} \mathbf{H}_{m} \mathbf{W}_{m} \mathbf{X}_{m}+\mathbf{v}
$$

with

$$
\begin{aligned}
& \mathbf{H}_{m}=\left[\mathbf{h}_{m, 1}, \cdots, \mathbf{h}_{m, \mathrm{U}_{\mathrm{k}}}\right], \\
& \mathbf{W}_{m}=\operatorname{diag}\left\{w_{m, 1}, \cdots, w_{m, \mathrm{U}_{\mathrm{k}}}\right\} \\
& \mathbf{x}_{m}=\left[x_{m, 1}, \cdots, x_{m, \mathrm{U}_{k}}\right]^{T},
\end{aligned}
$$

where $\mathbf{H}_{m}$ and $\mathbf{W}_{m}$ are the channel gain matrix and transmit power coefficient matrix of the $m$-th group, respectively. Besides, $\mathbf{x}_{m}$ denotes the transmit signal of users in the $m$-th group.

\subsection{Analysis of Achievable Sum Rate}

In light of power-domain NOMA, the order of demodulation is determined by the signal strength and priority is given to the users with higher power. Therefore, in the cell-free massive MIMO-NOMA system based on GSIC, since the equivalent path loss in the 1st group is the smallest, we firstly detect the user signals in the 1st group. Before the user signals in the 2nd group are decoded, all user signals in the 1st group will be removed, and so on, until the user signals in all groups are demodulated. The comparison between the new framework of GSIC-NOMA and the tradition SIC-NOMA based on user clustering is shown in Fig.2.

comparison

Figure 2 Comparison between GSIC-NOMA and SIC-NOMA.

As to the different groups, in the new framework, most of the inter-group interference can be removed by using GSIC. However, because of error propagation and the interference from undemodulated groups, there is still a few inter-group interference for the current demodulated group. Therefore, at the CPU, the all user signals in the $m$-th group can be expressed as

$$
\begin{aligned}
\hat{\mathbf{x}}_{m}= & \underbrace{\mathbf{E}_{\mathrm{m}}^{H} \mathbf{H}_{m} \mathbf{W}_{m} \mathbf{x}_{m}}_{[\text {Desired Signal] }}+\underbrace{\mathbf{E}_{\mathrm{m}}^{H} \sum_{i=m+1}^{M} \mathbf{H}_{i} \mathbf{W}_{i} \mathbf{x}_{i}}_{\begin{array}{c}
\text { [Inter-group } \\
\text { Interference after GSIC] }
\end{array}} \\
& +\underbrace{\mathbf{E}_{\mathrm{m}}^{H} \sum_{j=m-1}^{1} \mathbf{H}_{j} \mathbf{W}_{j} \boldsymbol{\Sigma}_{j} \mathbf{x}_{j}}_{\begin{array}{c}
\text { [Error Propagation } \\
\text { caused by Imperfect GSIC] }
\end{array}}+\underbrace{\mathbf{E}_{\mathrm{m}}^{H} \mathbf{v}}_{[\text {Noise] }}
\end{aligned}
$$

where $\mathbf{E}_{m}$ denotes the equalizer at the CPU for the users in the $m$-th group and $\boldsymbol{\Sigma}_{j}$ is a diagonal matrix, representing the interference of $j$-th group due to error 
propagation, which can be written as

$$
\boldsymbol{\Sigma}_{j}=\operatorname{diag}\left\{\sqrt{\varepsilon_{j, 1}}, \cdots, \sqrt{\varepsilon_{j, \mathrm{U}_{j}}}\right\}
$$

As to the different users in each group, in the new framework, they are distinguished by their respective equalizer $\mathbf{E}_{m}$. In addition to the inter-group interference, there is still the interference between different users within a group, so the signal of $\mathrm{UE}_{\mathrm{m}, \mathrm{k}}$ can be expressed as

$$
\begin{aligned}
& \hat{x}_{m, k}=\underbrace{\left[\mathbf{E}_{m}\right]_{:, k}^{H}\left[\mathbf{H}_{m}\right]_{:, k} w_{m, k} x_{m, k}}_{\text {Desired Signal }}+\underbrace{\left[\mathbf{E}_{m}\right]_{:, k}^{H} \sum_{i=m+1}^{M} \mathbf{H}_{i} \mathbf{W}_{i} \mathbf{x}_{i}}_{\begin{array}{c}
\text { [Inter-group } \\
\text { Interference after GSIC }]
\end{array}} \\
& +\underbrace{\left[\mathbf{E}_{m}\right]_{:, k}^{H} \sum_{j=m-1}^{1} \mathbf{H}_{j} \mathbf{W}_{j} \boldsymbol{\Sigma}_{j} \mathbf{x}_{j}}_{\begin{array}{c}
\text { [Error Propagation } \\
\text { camsed by Imperfect GSIC] }
\end{array}}+\underbrace{\left[\mathbf{E}_{m}\right]_{:, k}^{H} \mathbf{v}}_{\text {Noise }}
\end{aligned}
$$

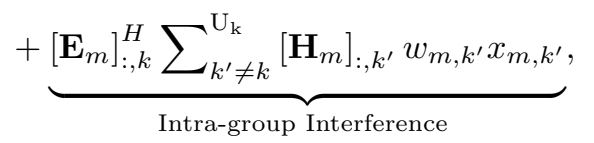

To facilitate the power control constraints in the next subsection, by using (12), an achievable sum rate for $\mathrm{UE}_{\mathrm{m}, \mathrm{k}}$ can be expressed as follows [7]:

$$
\begin{aligned}
R_{m, k} & =\log \left(1+\operatorname{SINR}_{m, k}\right) \\
& =\log \left(1+P_{d} /\left(\sum_{i=1}^{3} P_{I_{i}}+\sigma_{m, k}^{2}\right)\right)
\end{aligned}
$$

where $\mathrm{SINR}_{m, k}$ is the signal to interference-plus-noise ratio (SINR) of $\mathrm{UE}_{\mathrm{m}, \mathrm{k}}$, it reflects the performance of users. Besides, $P_{d}$ and $P_{I_{i}}(\mathrm{i}=1,2,3)$ are the power of desired signal and interference signals, respectively, which can be expressed as follows:

$$
\begin{aligned}
& P_{d}=\left|\left[\mathbf{E}_{m}\right]_{:, k}^{H}\left[\mathbf{H}_{m}\right]_{:, k} w_{m, k}\right|^{2}, \\
& P_{I_{1}}=\left[\mathbf{E}_{m}\right]_{:, k}^{H} \sum_{i=m+1}^{M} \mathbf{H}_{i} \mathbf{W}_{i} \mathbf{W}_{i}^{H} \mathbf{H}_{i}^{H}\left[\mathbf{E}_{m}\right]_{:, k}, \\
& P_{I_{2}}=\left[\mathbf{E}_{m}\right]_{:, k}^{H} \sum_{j=m-1}^{1} \mathbf{H}_{j} \mathbf{W}_{j} \boldsymbol{\Sigma}_{j} \boldsymbol{\Sigma}_{j}^{H} \mathbf{W}_{j}^{H} \mathbf{H}_{j}^{H}\left[\mathbf{E}_{m}\right]_{:, k}, \\
& P_{I_{3}}=\sum_{k^{\prime}=1, k^{\prime} \neq k}^{\mathrm{U}_{\mathrm{k}}}\left|\left[\mathbf{E}_{m}\right]_{:, k}^{H}\left[\mathbf{H}_{m}\right]_{:, k^{\prime}} w_{m, k^{\prime}}\right|^{2},
\end{aligned}
$$

By calculating per user's achievable sum rate, the achievable sum rate of the uplink cell-free massive MIMO-NOMA system based on GSIC can be written as

$$
R=\sum_{m=1}^{M} \sum_{k=1}^{\mathrm{U}_{\mathrm{k}}} R_{m, k}=\sum_{m=1}^{M} \sum_{k=1}^{\mathrm{U}_{\mathrm{k}}} \log _{2}\left(1+\mathrm{SINR}_{m, k}\right)
$$




\subsection{Power Control Scheme}

Many attentions have been paid to energy efficient communication in future wireless communication, so it is worth studying the total power consumption of the system. In this paper, we optimize the power control scheme, subjecting to per user's QoS and the maximum transmission power limit. Therefore, in the cell-free massive MIMO-NOMA system, the optimization formula of the total power consumption can be expressed as

$$
\begin{aligned}
\mathbf{P 1}: \min _{\left\{w_{m, k}\right\},\left\{\mathbf{E}_{m}\right\}}: & \sum_{m=1}^{M} \sum_{k=1}^{\mathrm{U}_{\mathrm{k}}} w_{m, k}^{2} \\
\text { s.t. } & \log _{2}\left(1+\mathrm{SINR}_{m, k}\right) \geq R_{m, k}, \\
& w_{m, k}^{2} \leq P_{\max },
\end{aligned}
$$

where $R_{m, k}$ and $P_{\max }$ represent the minimum transmission rate and the maximum transmission power of $\mathrm{UE}_{\mathrm{m}, \mathrm{k}}$, respectively. Since the 1st constraint in the optimization is non-convex, the problem $(\mathbf{P 1})$ is non-convex.

To simplify the non-convex optimization problem $(\mathbf{P 1})$, we use the relationship between the equalizer and the transmit power. To maximize per user's SINR, in[12], the best linaer equalizer is the minimum mean squre error (MMSE) equalizer. Therefore, we use the MMSE equalizer to demodulate the user signals in the $m$-th group, which can be expressed as

$$
\begin{aligned}
& \mathbf{E}_{m}=\left(\mathbf{G}_{m} \mathbf{X}_{m} \mathbf{X}_{m}^{H} \mathbf{G}_{m}^{H}+\sum_{i=m+1}^{M} \mathbf{H}_{i} \mathbf{W}_{i} \mathbf{W}_{i}^{H} \mathbf{H}_{i}^{H}\right. \\
& \left.+\sum_{j=m-1}^{1} \mathbf{H}_{j} \mathbf{W}_{j} \boldsymbol{\Sigma}_{j} \boldsymbol{\Sigma}_{j}^{H} \mathbf{W}_{j}^{H} \mathbf{H}_{j}^{H}+\sigma_{m, k}^{2} \mathbf{I}\right)^{-1} \mathbf{H}_{m} \mathbf{W}_{m}
\end{aligned}
$$

By substituting (20) into $\operatorname{SINR}_{m, k}$ and using Shannon formula, we can obtain per user's transmit power, which is expressed as (21).

$$
\begin{aligned}
& w_{m, k}^{2}=\left(2^{R_{m, k}}-1\right) /\left([ \mathbf { H } _ { m } ] _ { : , k } ^ { H } \left(\sum_{k^{\prime}=1, k^{\prime} \neq k}^{U_{k}}\left[\mathbf{H}_{m}\right]_{:, k^{\prime}}^{H}\left[\mathbf{H}_{m}\right]_{:, k^{\prime}} w_{m, k^{\prime}}^{2}\right.\right. \\
& \left.\left.+\sum_{i=m+1}^{M} \mathbf{H}_{i} \mathbf{W}_{i} \mathbf{W}_{i}^{H} \mathbf{H}_{i}^{H}+\sum_{j=m-1}^{1} \mathbf{H}_{j} \mathbf{W}_{j} \boldsymbol{\Sigma}_{j} \boldsymbol{\Sigma}_{j}^{H} \mathbf{W}_{j}^{H} \mathbf{H}_{j}^{H}+\sigma_{v}^{2} \mathbf{I}\right)^{-1}\left[\mathbf{H}_{m}\right]_{:, k}\right)
\end{aligned}
$$

Therefore, the original non-convex optimization problem $(\mathbf{P 1})$ is transformed to

$$
\begin{aligned}
\mathbf{P 2}: \min _{\left\{w_{m, k}\right\}}: & \sum_{m=1}^{M} \sum_{k=1}^{\mathrm{U}_{\mathrm{k}}} w_{m, k}^{2} \\
\text { s.t. } & \log _{2}\left(1+\mathrm{SINR}_{m, k}\right) \geq R_{m, k}, \\
& w_{m, k}^{2} \leq P_{\max },
\end{aligned}
$$

After the transformation, the original joint power control and equalizer optimization problem is transformed to a pure power control problem. We use a parallel iteration method to achieve the best power control scheme. The so-called parallel 
iteration refers to obtaining the minimum power consumption of the system through multiple internal iteration and external iteration. Internal iteration refers to user iteration within a group. In the case of fixed inter-group interference, the internal user power within a group is updated according to (21). External iteration refers to user iteration between all groups. After all groups complete an internal iteration, an external iteration is performed to determine whether the total power consumption of the system has converged. If it converges, it is considered that the total power consumption of the system is the lowest at this time. The detailed procedure is summarized in Algorithm 1.

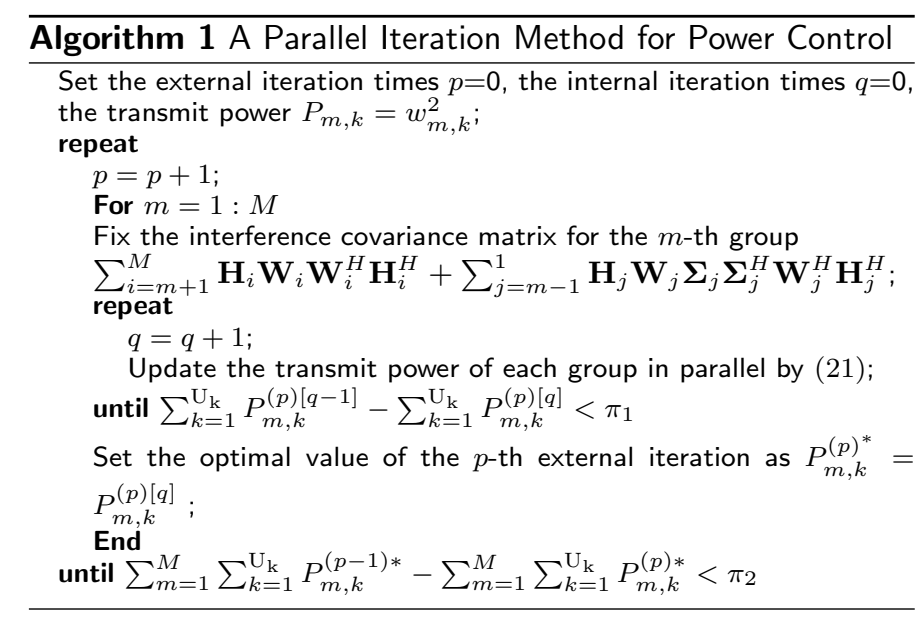

\section{Results and Discussion}

In thie section, we provide simulation results to assess the performance of cell-free massive MIMO-NOMA based on GSIC and compare it with OMA and SIC-NOMA based on user clustering, respectively. We consider that the $L$ APs and $M$ users with single-antenna are uniformly and randomly distributed in a square of $\mathrm{D} \times \mathrm{Dm}^{2}$. In this paper, calculation method of large-scale channel gain $\beta_{l, m, k}$ is found in [10]. Other simulation parameters are presented in Table 1.

Table 1 Simulation Parameters

\begin{tabular}{cc}
\hline Parameters & Value \\
\hline The noise power & $-99 \mathrm{dBm}$ \\
The length of square $D$ & $500 \mathrm{~m}$ \\
The number of groups $M$ & 2 \\
The error propagation factor $\varepsilon_{m, k}$ & 0.01 \\
The number of users in a group $\mathrm{U}_{\mathrm{k}}$ & 50 \\
The termination thresholds $\pi_{1}, \pi_{2}$ & $10^{-4}$ \\
The maximum transmit power $P_{\max }$ & $20 \mathrm{dBm}$ \\
The minimum rate requirement $R_{m, k}$ & $0.1 \sim 0.5$ \\
\hline
\end{tabular}

First of all, the effect of the minimum rate requirement for the users in a group on the total transmit power consumption is investigated in this paper. Fig. 3 depicts numerically user rate requirement versus the total transmit power consumption in the cell-free massive MIMO-NOMA system, for $K=100, L=50, M=2$. It is shown that the total power consumption is increased with user rate requirement increasing. Compared with OMA and the traditional SIC-NOMA based on user clustering, the proposed scheme has obvious advantages in total power consumption. The performance of the traditional SIC-NOMA is the worst. It is because that 
the user-clustered method will lead the equivalent channel gain between some users and AP to be quite small after beamforming, therefore, the system's transmit power consumption is high. In the same time slot, compared with OMA, the proposed scheme in this paper enables to separate twice as many users than OMA. Besides, it removes inter-group interference through GSIC, which effectively reduces inter-user interference and improves the SINR of the target user, therefore the total power consumption of the system is reduced.

simulation1

Figure 3 The total transmit power versus the minimum rate

Next, the impact of the number of APs on the total transmit power consumption is evaluated. Under the condition that the number of users and the number of AP$\mathrm{s}$ both increase in proportion, Fig. 4 shows the total transmit power for different numbers of APs, with $R_{1, k}=0.1 \mathrm{bit} / \mathrm{s} / \mathrm{Hz}, M=2$. It can be seen from the figure that when the GSIC-NOMA scheme and OMA scheme are used for transmission, as the number of APs increases, the total transmit power consumption of the system decreases accordingly. The reason is that when the number of APs increases, multiple users can be better distinguished during the period of demodulation as the interference between users is reduced effectively. Therefore, the total transmit power consumption of the system is reduced. However, for the traditional SIC-NOMA based on user clustering, this benefit fades away, the interference between users increases sharply as the number of users increases, which causes the total transmit power consumption of the system to increase drastically.

simulation2

Figure 4 The total transmit power versus the number of APs

\section{Conclusion}

In this paper, the performance of uplink cell-free massive MIMO-NOMA system based on GSIC has been investigated. A new method of user grouping according to per user's equivalent path loss is put forward to combine with GSIC. The intergroup users' distinguishing follows the principle of NOMA, while the principle of SDMA is invoked during the intra-group transmission. This scheme realizes transmission with deep integration between MIMO and NOMA. What's more, we use the relationship between the equalizer and the transmit power coefficient to transform the power optimization problem, and a parallel iterative method is used to obtain the optimal power control of the system. The simulation results show that, under the consideration of error propagation, compared with OMA and the traditional SIC-NOMA based on user clustering, the proposed scheme can effectively reduce the total transmit power consumption of the system. It is worth mentioning that the effect of the GSIC-NOMA is more remarkable when the number of users is large. 


\section{Abbreviations}

NOMA: Non-orthogonal multiple access; MIMO: Multiple-input multiple-output; GSIC: Group-level successive interference cancellation; OMA: Orthogonal multiple access; CSI: Channel state information; SIC: Successive interference cancellation; SDMA: Space division multiplex access; RIS: Reflecting intelligent surface; AP: Access point; SINR: Signal to interference-plus-noise ratio; MMSE: Minimum mean squre error.

Acknowledgements

The authors would like to thank NJUPT for their support and any one who supported the publication of this paper.

Authors' contributions

All authors have contributed equally. All authors read and approved the final manuscript.

Funding

This work was supported by the National Natural Science Foundation of China (NO. 621712335), and by the open research fund of National Mobile Communications Research Laboratory, Southeast University (NO. 2021D10).

\section{Availability of data and materials}

Not applicable

Ethics approval and consent to participate

Ethical approval.

\section{Competing interests}

The authors declare that they have no competing interests.

Consent for publication

Not applicable

\section{Author details}

${ }^{1}$ Key Lab of Broadband Wireless Communication and Sensor Network Technology, Nanjing University of Posts and Telecommunications, Nanjing, China. ${ }^{2}$ National Mobile Communications Research Laboratory, Southeast University, Nanjing, China.

\section{References}

1. Chen, Y., Bayesteh, A., Wu, Y., Ren, B., Kang, S., Sun, S., Xiong, Q., Qian, C., Yu, B., Ding, Z., Wang, S., Han, S., Hou, X., Lin, H., Visoz, R., Razavi, R.: Toward the Standardization of Non-Orthogonal Multiple Access for Next Generation Wireless Networks. IEEE Commun. Mag. 56(3), 19-27 (2018). doi:10.1109/MCOM.2018.1700845

2. Islam, S.M.R., Avazov, N., Dobre, O.A., Kwak, K.-s.: Power-Domain Non-Orthogonal Multiple Access (NOMA) in 5G Systems: Potentials and Challenges. IEEE Commun. Surv. Tutor. 19(2), 721-742 (2017). doi:10.1109/COMST.2016.2621116

3. Ding, Z., Schober, R., Poor, H.V.: A General MIMO Framework for NOMA Downlink and Uplink Transmission Based on Signal Alignment. IEEE Trans. Wirel. Commun. 15(6), 4438-4454 (2016). doi:10.1109/TWC 2016.2542066

4. Huang, Y., Zhang, C., Wang, J., Jing, Y., Yang, L., You, X.: Signal Processing for MIMO-NOMA: Present and Future Challenges. IEEE Wirel. Commun. 25(2), 32-38 (2018). doi:10.1109/MWC.2018.1700108

5. Wang, H., Zhang, R., Song, R., Leung, S.-H.: A Novel Power Minimization Precoding Scheme for MIMO-NOMA Uplink Systems. IEEE Commun. Lett. 22(5), 1106-1109 (2018). doi:10.1109/LCOMM.2018.2812786

6. Wang, H., Liu, C., Shi, Z., Fu, Y., Song, R.: GSIC for RIS-Aided Uplink Multi-Antenna NOMA Systems. IEEE Commun. Lett. 26(1), 187-191 (2022). doi:10.1109/LCOMM.2021.3125689

7. Ngo, H.Q., Ashikhmin, A., Yang, H., Larsson, E.G., Marzetta, T.L.: Cell-Free Massive MIMO Versus Small Cells. IEEE Trans. Wirel. Commun. 16(3), 1834-1850 (2017). doi:10.1109/TWC.2017.2655515

8. Zhang, J., Chen, S., Lin, Y., Zheng, J., Ai, B., Hanzo, L.: Cell-Free Massive MIMO: A New Next-Generation Paradigm. IEEE Access 7, 99878-99888 (2019). doi:10.1109/ACCESS.2019.2930208

9. Li, Y., Aruma Baduge, G.A.: NOMA-Aided Cell-Free Massive MIMO Systems. IEEE Commun. Lett. 7(6), 950-953 (2018). doi:10.1109/LWC.2018.2841375

10. Rezaei, F., Heidarpour, A.R., Tellambura, C., Tadaion, A.: Underlaid Spectrum Sharing for Cell-Free Massive MIMO-NOMA. IEEE Commun. Lett. 24(4), 907-911 (2020). doi:10.1109/LCOMM.2020.2966195

11. Zhang, Y., Cao, H., Zhou, M., Yang, L.: Spectral Efficiency Maximization for Uplink Cell-Free Massive MIMO-NOMA Networks. In: 2019 IEEE International Conference on Communications Workshops (ICC Workshops), pp. 1-6 (2019). doi:10.1109/ICCW.2019.8756881

12. Wang, H., Song, R., Leung, S.-H.: Mitigation of uplink ICI and IBI in OFDMA two-tier networks. IEEE Trans. Veh. Technol. 65(8), 6244-6258 (2015)

\section{Additional Files}

Additional file 1 - DGX

DGX is Fig.1, it is displayed in folder in the form of EPS.

Additional file 2 - comparison

comparison is Fig.2, it is displayed in folder in the form of EPS.

Additional file 3 - simulation1

simulation1 is Fig.3, it is displayed in folder in the form of EPS. 
Additional file $4-$ simulation2

simulation2 is Fig.4, it is displayed in folder in the form of EPS. 
Figures

\section{Figure 1}

Ilustration of equivalent path loss contour for a cell-free system with ve APs.

\section{Figure 2}

Comparison between GSIC-NOMA and SIC-NOMA.

\section{Figure 3}

The total transmit power versus the minimum rate 


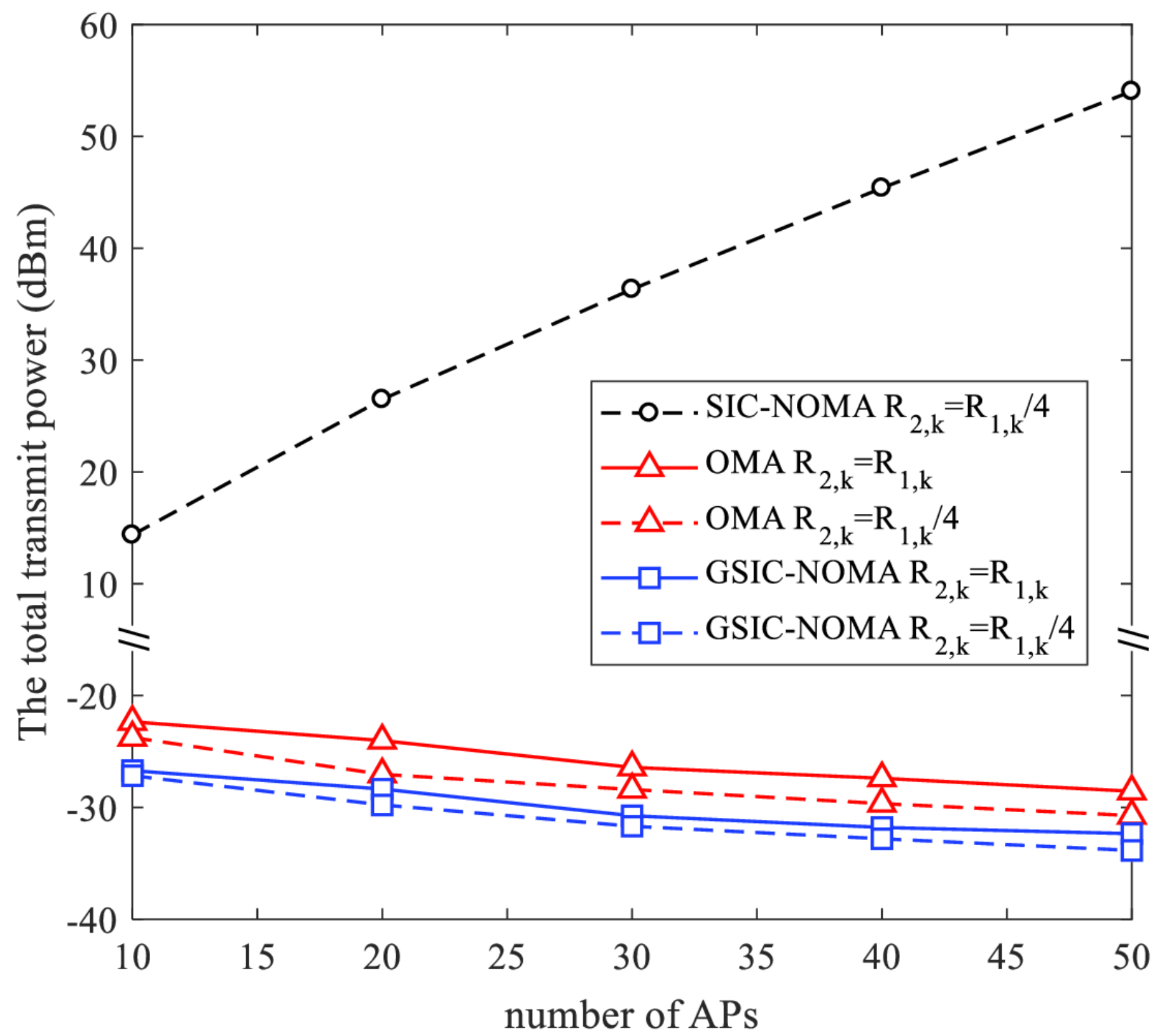

Figure 4

The total transmit power versus the number of APs 